\title{
SWIMMING AGAINST THE FLOW: A ROBOTICS SIMULATION FRAMEWORK
}

\section{S. Cecilia Tapia Siles and Ryad Chellali}

\begin{abstract}
Fish in nature take advantage of some types of turbulence and even generate it to swim with a minimum expenditure of energy. This is the case observed in rainbow trout swimming against the flow in well patterned turbulence phenomenon called Karman Street.

Robotic and Multiphysics simulators do not include the possibility of this sort of turbulent flow in interaction with the robot body, to train controllers. Therefore, to better understand how to design a robot that takes advantage of the turbulence, we have developed a simulation framework based on rigid body dynamics software (Webots) and a physics plugin. This plugin has been developed based on a generalized abstraction in the useful area of Karman vortex streets.

This framework allows the simulation of user designed robots and their controller interaction with the environment.
\end{abstract}

Keywords: Underwater Robotics, Turbulence, Energy Harvesting, Simulation. 